\title{
Literaturhinweise
}

\author{
Zur grundlegenden Literatur über den Verlag siehe die Übersicht auf S. 15.
}

\section{Konturen des Anfangs}

Nördlingen: G.A. Zipperer, Nördlingen. Lebenslauf eines schwäbischen Stadt, 1979; D.H. Voges, Die Reichsstadt Nördlingen. 12 Kapitel aus ihrer Geschichte, 1988; Die C.H.Beck'sche Buchdruckerei Nördlingen in den Jahren 1945-1979, 1980, S. I f. Der Vergleich mit «Kuhschnappel» bei $M$. Stolleis, Der Aquädukt 1763-1988. Ein Almanach aus dem Verlag C.H.Beck, 1988, S.22 ff. W.L. Wekhrlin in seiner Zeitschrift «Das Graue Ungeheuer», 4. Band, 1784 (Ndr. 1976), S. 252 f. Buchhandel: F. Kapp, Geschichte des Deutschen Buchhandels, Bd. 1, 1886, S. 448 ff. (Frankfurter Messe); J. Goldfriedrich, Geschichte des Deutschen Buchhandels, Bd. 2, 1908. S. 136 ff. (Leipziger Messe, Tauschhandel, das Zitat S. 271), Bd. 3, 1909, S. 185 ff. (Nettohandel, Konditionssystem, Nachdrucke); G. Menz, Der deutsche Buchhandel, 1925, S. 72 ff. (ab Tauschhandel, das Zitat S. 94, f.); R. Wittmann, Geschichte des deutschen Buchhandels, 1999, S. 82 ff. (ab Tauschhandel, das Zitat S. 102); die Anzeige Carl Gottlob Becks von 1775 bei: B. Hampp, Wilhelm Ludwig Wekhrlin und Karl Gottlob Beck. Ein Publizist und sein Verleger zur Zeit der Aufklärung, Diplomarbeit der Univ. Eichstätt, 2oo1, S. 59. Zum Beckschen Antiquariat: Oscar Beck, 1913 , S. $39 \mathrm{f}$.

\section{Carl Gottlob, Luise, Carl Heinrich und Catharina Magdalena Beck}

Carl Gottlob Beck: $R$ Schmidt, Deutsche Buchhändler. Deutsche Buchdrucker, 1912 (Ndr.1979) S. 35 ff.; Oscar Beck 1913, S. 13 ff.; Heinrich Beck, 1963, S. 1 ff.; B. Hampp, Wilhelm Ludwig Wekhrlin und Carl Gottlob Beck. Ein Publizist und sein Verleger zur Zeit der Aufklärung, Diplomarbeit Univ. Eichstätt, 2001, S. $5^{1}$ ff., dort S. 56 ff. zu den finanziellen Schwierigkeiten am Ende seines Lebens; der Lagerkatalog Nicolais 1787: P. Rabe, Zum Bild des Verlagswesens in Deutschland der Spätaufklärung. Dargestellt an Hand von Friedrich Nicolais Lagerkatalog 1787, in R. Wittmann u. B. Hack (Hg.), Buchhandel und Literatur, 1982, S. 152. Heinrich Christian Senckenberg: R. v. Stintzing, E. Landsberg, Geschichte der deutschen Rechtswissenschaft, Abt. 3. Halbbd.1, S. 245 ff.; Halbbd.2, S. 162 f., 1898 (Ndr.1978). Wilhelm Ludwig Wekhrlin: J. Mondot, Nachwort, in: Anselmus Rabiosus Reise durch Oberdeutschland, 1988, S. 135 ff.; B. Натpp a. a. O. S. 22 ff. Zu Carl Gottlob Beck und Wekhrlin: B. Hampp a. a. O. S. 61 ff.; Carl Gottlob Beck, Klage gegen Wilhelm Ludwig Wekhrlin, hg. v. J. Mondot, in: E.-P. Wiekenberg (Hg.), Einladung ins 18. Jahrhundert. Ein Almanach aus dem Verlag C.H.Beck, 1988, S. 379 ff. Zeitschriften der Aufklärung: $O$. Dann, Die Zeitschriften der deutschen Aufklärungsgesellschaft, in: M. Stolleis (Hg.), Juristische Zeitschriften - die Medien des 18.-2o. Jahrhunderts, 1999, S. 1 ff.; R. Stöber, Deutsche Pressegeschichte, 2. Aufl. 2oo5, S. 86 ff., zu Wekhrlin dort S.92. Georg Gottfried Strelin: A. Schwennicke, «Ohne Steuer kein Staat». Zur Entwicklung und Funktion des Steuerrechts in den Territorien des Heiligen Römischen Reichs (1500-180o), 1996, S. 341 f. Carl Heinrich Beck: Oscar Beck 1913, S. 31 ff; Antiquariat: Heinrich Beck, 1969, S.16; Wirtschaft Nördlingens um ı80o: G. A. Zipperer, Nördlingen. Lebenslauf einer schwäbischen Stadt, 1979, S.19o ff.- Catharina Magdalena Beck: 
Oscar Beck 1913, S.33ff; Johann Caspar Bluntschli: J.P. Arquint, Bluntschli, Johann Kaspar, in: M. Stolleis (Hg.), Juristen, 1995, S.95 mit weiterer Literatur; zu Johann Caspar Bluntschli (und auch Friedrich Rohmer): E. Forster, Johann Caspar Bluntschli, in: G. Kleinheyer, J. Schröder (Hg.), Deutsche und Europäische Juristen aus neun Jahrhunderten, 5. Aufl. 2oo8, S. 7o f., dort auch zu der Blamage mit Friedrich Rohmer in Zürich. S. 33 ff.; das Zitat zu Georg Friedrich Puchta: Ernst Landsberg, Geschichte der deutschen Rechtswissenschaft, Abt. 3, Halbbd. 2, 1910 (Ndr. 1978), S. 439 f.

\section{Märzrevolution und Reichsgründung}

H. Lutz, Zwischen Habsburg und Preußen. Deutschland 1815-1866, 1985; M. Stürmer, Das ruhelose Reich. Deutschland 1866-1918, 1983 T. Nipperdey, Deutsche Geschichte 1866-1918, 2.Bd., 1992, das Zitat S. 202 f. H. Kessler, Politische Bewegungen in Nördlingen und dem bayerischen Ries während der Deutschen Revolution 1848/49, 1939 .

\section{Carl und Eugenie Beck, Ernst Rohmer als Statthalter für die Söhne Carl Becks}

Carl Beck: Oscar Beck, Verlagskatalog der C.H.Beck'schen Verlagsbuchhandlung, 17631913, 1913, Geschichtliche Einleitung, S. 35 ff.; H. Kessler, Politische Bewegungen in Nördlingen und dem bayerischen Ries während der deutschen Revolution 1848/49, 1939, S. 83, 96, 179, 191 f. (dort die Zitate aus den Akten der Augsburger Regierung), 279. Volksverein und Nördlingen allgemein: $H$. Kessler S. 96 ff., 245, 277. Karl Brater: $H$. Kessler S. 169 ff.; A. Sapper (seine Tochter), Frau Pauline Brater, 1908, auch zu seinen finanziellen Schwierigkeiten nach 1851; T. Schieder, Brater, Karl, in: Neue Deutsche Biographie, Bd. 2, S. 538 mit weiterer Literatur. Blätter für die administrative Praxis: das Zitat Braters in Bd. 1, S.4; M. Stolleis, Geschichte des öffentlichen Rechts in Deutschland, Bd. 2, 1992, S. 285; C. Doerfert, Die Zeitschriften des öffentlichen Rechts 1848-1933, in: M. Stolleis (Hg.), Juristische Zeitschriften. Die neuen Medien des 18.-2o. Jahrhunderts, 1999, S. 421 ff.; H. Weber, Juristische Zeitschriften des Verlages C.H.Beck, 2007, S. 2 ff. Eugenie Beck: Oscar Beck 1913, S. 38, 44, 69 ff. Ernst Rohmer: Oscar Beck a. a. O S. 45 ff.; Artikel: Rohmer, Ernst, in: Neue Deutsche Biographie, Bd. 22, 2005, S. I f. Die Zeitschrift für das Notariat: Hermann Weber 2007, S. 5 f. Die juristische Buchproduktion zur Zeit Ernst Rohmers: G. Jäger, Juristischer Verlag, in: G. Jäger u. a. (Hg.), Geschichte des deutschen Buchhandels im 19. und 20. Jahrhundert, Bd. 1, Das Kaiserreich 1870-1918, Teil 1, 2001, S. 486. Zahlen über Buchproduktion und Sachbereiche des Verlags von Carl Beck bis Ernst Rohmer nach Durchsicht des Verlagskatalogs 1763-1913 bei Oscar Beck 1913, S. 157 ff.

\section{Kaiserreich, Weimarer Republik und Buchhandel}

M. Stürmer, Das ruhelose Reich. Deutschland 1866-1918, 1983; H. Schulze, Weimar. Deutschland 1917-1933, 1982; H.A. Winkler, Weimar 1918-1933, 1993. «Gründerkrise»: T. Nipperdey, Deutsche Geschichte 1866-1918, Bd.1, 1990, S.283 ff. und R. Walter, Wirtschaftsgeschichte, 4.Aufl. 2003, S.105 ff. Buchhandel bis 1918: R. Wittmann, Geschichte des deutschen Buchhandels, erw. Aufl. 1999, S.257 ff.; M. Esterwegen, G. Jäger, Geschichtliche Grundlagen und Entwicklung des Buchhandels im Deutschen Reich bis 1871, in: G. Jäger (Hg.), Geschichte des deutschen Buchhandels im 19. und 2o. Jahrhundert, Bd.1, Das Kaiserreich 1870-1918, 2001, S. 17 ff. (auch zur 1. Leserevolution); zum wissenschaftlichen und juristischen Verlag dort G. Jäger S.423 ff. und 486 ff. Zu den Ursachen der Stagnation seit 1848: J. Goldfriedrich, Geschichte des Deutschen Buchhandels, 4.Bd.; 1913, S.zo1 ff. und R. Wittmann a. a. O. S.257 ff., dort S.265 zur Krönerschen Reform. Buchhandel in der Weimarer Zeit: B. Kastner, Statistik und Topographie des Ver- 
lagswesens, in: E. Fischer, S. Füssel (Hg.), Geschichte des deutschen Buchhandels im 19. und 20. Jahrhundert. Die Weimarer Republik 1918-1933, Teil 1, 2007, S. 341 ff.; U. Schneider, Der wissenschaftliche Verlag, in: E. Fischer, S. Füssel a. a. O. S. 379 ff.; zur «Bücherkrise»: E. Fischer, Marktorganisation, in: E. Fischer, S. Füssel a. a. O. S. 273 ff., dort S. 274 das Zitat Samuel Fischers.

\section{Oscar Beck}

Oscar Beck: Heinrich Beck 1963, S. 57 ff.; W. Zorn, Lebensbilder aus dem Bayerischen Schwaben, 1966, S. $258 \mathrm{ff}$. Die juristische Buchproduktion zur Zeit Oscar Becks: $G$. Jäger, wie Lit. zu VIII, dort S. 498 ff. zum Verlag C.H.Beck. Walter Flex: R. Neuß, Anmerkungen zu Walter Flex, 1992; H.R. Wahl, Die Religion des deutschen Nationalsozialismus, 2002 , S. 283 ff.; H. Wagener, Wandervogel und Flammengel. Walter Flex: Der Wanderer zwischen beiden Welten ..., in: T. F. Schneider, H. Wagener (Hg.), Von Richthofen bis Remarque, 2003, S. 17 ff. Johann Julius Planck: Hellmann, Johann Julius Wilhelm von Planck (Nachruf), in: Deutsche Juristen-Zeitung 1900, S. 409; der Nachdruck des Lehrbuchs 1970 im Keip Verlag Frankfurt am Main. Otto Fischer: H. Kiefner, Fischer, Otto, Jurist, in: Neue Deutsche Biographie, Bd. 5, 1961, S. 202 f.; S. Saar, Zwischen PreuBischen Privatrecht und BGB - Otto Fischer (1853-1929), in: Festschrift H. Kollhosser, 2004, Bd. 2, S. 202 f. Glossatoren und Kommentatoren: F. Wieacker, Privatrechtsgeschichte der Neuzeit, 2. Aufl., 1967, S. 45 ff., 8 o ff. (er nennt die Kommentatoren noch Konsiliatoren); H. Schlosser, Grundzüge der Neueren Privatrechtsgeschichte, 1o. Aufl., 2005, S. 36 ff.; U. Wesel, Geschichte des Rechts, 3 . Aufl. 2006, S. 317 ff. Carl Sartorius: Heinrich Beck 1963, S. 165; M. Stolleis, Geschichte des öffentlichen Rechts in Deutschland, Bd.3, 1999, S. 291, dort in Anm. 291 die Unsterblichkeit; M. Otto, Sartorius, Carl, in: Neue Deutsche Biographie, Bd.22, 2005, S. 440 f. Fritz Stier-Somlo: P. Landau, Juristen jüdischer Herkunft im Kaiserreich und in der Weimarer Republik, in: H. Heinrich, H. Franzki, K. Schmalz, M. Stolleis (Hg.), Deutsche Juristen jüdischer Herkunft, 1993, S. 181

\section{Schwabing}

K. Bäthe, Wer wohnte wo in Schwabing? 1965; H. Bauer, E. Tworek, Schwabing. Kunst und Leben um 1900, 1998, darin S. 159 ff. R. Wittmann, Verlage in Schwabing; G. Brauer, München Schwabing. Ein Zustand, 2010. G. Fürmetz (Hg.), Schwabinger Krawalle, 2006.

\section{Heinrich Beck in der Weimarer Republik}

1. Heinrich Beck: Heinrich Beck 1963, S. 167 ff.; Hans Dieter Beck 1988, S. 25 ff.; Wolfgang Beck 1988, S. XXVI ff. München 1918/19: A. Mitchell, Revolution in Bayern 1918/19, 1967 . Inflation und Rentenmark: R. Walter, Wirtschaftsgeschichte, 5. Aufl. 2011, S. $161 \mathrm{ff}$. Spengler: A. M. Kotanek, Oswald Spengler in seiner Zeit, 1968; D. Felken, Oswald Spengler, 1988. Friedrich Reck, Tagebuch eines Verzweifelten, 1981 (DIE ANDERE BIBLIOTHEK), S. 6 zur Ermordung Willi Schmids: B. Lahusen, Um jeden Preis, in: Lettre International, Heft 85, 2009, S. 87 ff.; 1935 erschien von Willi Schmid eine posthume Sammlung seiner Schriften mit einem Beitrag Oswald Spenglers: Willi Schmid, Unvollendete Symphonie, 1935. Schweitzer: Heinrich Beck 1963, S.115ff.; der Brief von 1962: Der Aquädukt 1988, S. $524 \mathrm{f}$.

2. Friedell: $W$. Lorenz, Egon Friedell, 1994. Die juristische Titelproduktion 1919-1933: B. Kastner, Statistik und Topographie des Verlagswesens, in: E. Fischer, S. Füssel (Hg.), Geschichte des deutschen Buchhandels im 19. und 2o. Jahrhundert. Die Weimarer Republik 1918-1933, Teil 1, 2007, S.344. Fritz van Calker: Wikipedia, Fritz van Calker. Glungler: M. Stolleis, Geschichte des öffentlichen Rechts in Deutschland, Bd. 3, 1999, 
S. 272 Anm. 169. Hans Frank: Stolleis a. a. O. S. 288 Anm. 272. Glungler und Frank werden beide genannt im Vorwort des Buchs van Calkers.

3. Schönfelder: H. Wrobel, Heinrich Schönfelder. Sammler Deutscher Gesetze (19021944), 1947; J. Wasmuth, Schönfelder, Deutsche Gesetze, in: D. Willoweit (Hg.), Rechtswissenschaft und Literatur im 20. Jahrhundert, 2007, S. 433 ff. Zu Mazdaznan/Masdasnan: U. Linse, Mazdaznan, in: H. Baer u. a. (Hg.), Lexikon neureligiöser Gruppen, Szenen und Weltanschauungen, 2005, Sp. 774 ff. Das Zitat aus der Rezension des Vorsitzenden des Justizprüfungsamts Berlin: H. David, Dr. Heinrich Schönfelder, Prüfe dein Wissen, Juristische Wochenschrift 1929, S. 1868 f.; zur Änderung des juristischen Unterrichts und des Referendarexamens: $W$. Kunkel, Die Juristenausbildung in der Zeit von 19341945, in: Veröffentlichungen des Arbeitskreises für Fragen der Juristenausbildung e.V., Die Ausbildung der deutschen Juristen, 1960, S. 70 f.

4. Der Greif: C.A. v. Volborth, Fabelwesen der Heraldik in Familien und Städtewappen, 1996, S. $4_{2}$ ff.; H. Gebhardt, M. Klug, Von Drachen, Yetis und Vampiren, 2005, S. $78 \mathrm{ff}$. Wappen, Heraldik: C.-A. v.Volborth, Heraldik, 1989 ; V.V.Filip, Einführung in die Heraldik, 20oo. Schilde der Germanen: Tacitus, Germania 6.ı. Wappen der Buchdrucker: E. Dilba, Typographie-Lexikon, 2. Aufl. 2008, S.26 Druckermarken: H. Wendland, Druckermarke, in: S. Corsten u. a. (Hg.), Lexikon des gesamten Buchwesens, Bd.2, 2. Aufl. 1989, S. 367. Entwicklung des Markenwesens und Markenschutzes: E. Leitherer, Die Entwicklung des Markenwesens, Diss. 1954, Ndr. 1988; E. Wadle, Fabrikzeichenschutz und Markenrecht, 2 Teile, 1977/83 (19. Jh.); E. Wadle, Geistiges Eigentum, Bd.1, 1996. Das Markengesetz von 1995: Schönfelder, Deutsche Gesetze, Nr. 72.

\section{Das «Dritte Reich» und der Buchhandel}

H.-U. Thamer, Verführung und Gewalt. Deutschland 1933-1945, 1986; M. Hirsch, D. Majer, J. Meinck, (Hg.), Recht, Verwaltung und Justiz im Nationalsozialismus, 2. Aufl. 1997; $R$. Wittmann, Geschichte des deutschen Buchhandels, 2. Aufl. 1999, S. 360 ff.

\section{Heinrich Beck im «Dritten Reich»}

1. Arisierungen: H. Genschel, Die Verdrängung der Juden aus der Wirtschaft im Dritten Reich, 1966; A. Barkai, Vom Boykott zur «Entjudung», 1988. Beiersdorf: F. Bajohr, «Arisierung» in Hamburg, 1998, S. 36 ff. Adolf Sommerfeld: $C$. Kress, Frühe «Arisierungen in der Bauindustrie: Adolf Sommerfeld und seine Firmengruppe, in: C. Biggeleben, B. Schreiber, K. J.L. Steiner (Hg.), «Arisierung in Berlin», 2007, S. 151 ff. Verlag Bensheimer: H. Göppinger, Juristen jüdischer Abstammung im «Dritten Reich», 199o, S. $376 \mathrm{f}$,, R. Schmidt, Deutsche Buchhändler, Deutsche Buchdrucker, 1902/1908, Ndr. 1979, S. 376 ff. Verlag J. Heß: Göppinger a. a. O. S. 376 f.; Schmidt a. a. O. S. 435 ff. Verlag Walther Rothschild: Göppinger a. a. O. S. $381 \mathrm{f}$.

2. Otto Liebmann: P. Ebel, O. Liebmann, in: R. Schmidt (Hg.), Deutsche Buchhändler. Deutsche Buchdrucker, Bd. 4, 1907 (Ndr. 1979), S.617 f; (wohl O.L. selbst) Liebmann, Otto, in: Reichshandbuch der Deutschen Gesellschaft, Bd. 2, 1930, S. 119 f. Deutsche Juristen-Zeitung: B. Rüthers, M. Schmitt, Die juristische Fachpresse nach der Machtergreifung der Nationalsozialisten, in: Juristenzeitung 1988, S. 370; M. Hachenburg. Lebenserinnerungen eines Rechtsanwalts und Briefe aus der Emigration, 1978 (die Erinnerungen zuerst 1927), S. 134ff, das Zitat S. 134, zur JW und Julius Magnus S. 136 f. Liliputausgaben: G. Jäger, Juristischer Verlag, in: G. Jäger u. a. (Hg.), Geschichte des Deutschen Buchhandels im 19. und 20. Jahrhundert. Das Kaiserreich 1870-1918, Teil 1, 2001, S. 487 f.; S. 502 f. zum Liebmann Verlag allgemein. Systematische Methode der Liebmann'schen Kommentare: Brief Liebmanns an Ebel im Besitz von Wolfgang Beck («Ebel-Notizen 47», vgl. unten 4.); Staub's Erfindung der systematischen Methode: H. Heinrichs, Hermann Staub (1856?-1904), in: H. Heinrichs u. a. (Hg.), Deutsche Juristen 
jüdischer Herkunft, 1993, S. 393 f.; Otto Liebmann in der DJZ 1904, Sp. 828. Adolf Baumbach: W. Hefermehl, Adolf Baumbach, in: Juristen im Portrait, S. 130 ff.; das Zitat Baumbachs in der Deutschen Richterzeitung 1928, Sp. 42 f. Ernst Strassmann: H. R. Sassin, Liberale im Widerstand. Die Robinsohn-Strassmann-Gruppe 1934-1942, 1993. Otto Schwarz: F.K. Kaul, Geschichte des Reichsgerichts, Bd.4, 1933-1945, 1971, S. 292 f.; Heinrich Beck 1963, S. 194. Leo Rosenberg: K. H. Schwab, Leo Rosenberg, in: Juristen im Portrait, S. 65 off. Marie Munk: M. Röwerkamp, Jristinnen, 2005, S. 275 ff. Tag von Potsdam: H.-v. Thamer, Verführung und Gewalt, Deutschland 1933-1945, 1986, S. 270 ff. Die Beerdigung Otto Liebmanns: H. Göppinger, Juristen jüdischer Abstammung im «Dritten Reich», 2. Aufl. 1990, S. 375 f.

3. Kauf des Verlags von Otto Liebmann: Heinrich Beck 1963, S. 17 off.; Hans Dieter Beck 1988, S. 27 f.; Wolfgang Beck 1988, S.XXXI f.; D. Willoweit, Das Profil des Verlages C.H.Beck im 2o. Jahrhundert, in: Rechtswissenschaft und Rechtsliteratur, S. 67 ff.; $C$. Busse, Deutsche juristische Literatur des 2o. Jahrhunderts, in: Kritische Justiz 2o10, S. 319 ff. Ullstein-Verlag: $H$. Gerschel, Die Verdrängung der Juden aus der Wirtschaft im Dritten Reich, 1966, S. 128 Anm. 48a.

4. Der Verleger im «Dritten Reich»: Heinrich Beck 1963, S. 170 ff.; Hans Dieter Beck 1988, S. 27 ff.; Wolfgang Beck 1988, S. XXI ff. Heimito v. Doderer: Heinrich Beck 1963, S. 183, 256 ff. G. Lenotre: Heinrich Beck 1963, S. 184, f.; R. Constans, G. Lenotre, reporter du passé, in: La Nouvelle Revue d'Histoire, 2oo6, S. 18 ff. Die Affäre Friedell 1937: Bundesarchiv Berlin. Die juristischen Zeitschriften: Heinrich Beck 1963, S. 181, 172 f.; B. Rüthers, M. Schmitt, Die juristische Fachpresse nach der Machtergreifung der Nationalsozialisten, in: Juristenzeitung 1988, S. 369 ff., dort S. 370 ff. zur DJZ, S. 372 f. zur JW, S. 374 zum Widerstand bei Heymanns. H. Göppinger, Juristen jüdischer Abstammung im «Dritten Reich», 1990, S. 374 ff., ebenfalls zur JW und DJZ; L. Becker, Die «Selbstgleichschaltung» juristischer Zeitschriften im Nationalsozialismus, in: M. Stolleis (Hg.), Juristische Zeitschriften, 1999, S. 481 ff.; zu den Beck'schen Zeitschriften zuletzt $C$. Busse, Deutsche juristische Literatur. Annotierte Rezension zweier Beschreibungen aus dem Hause Beck, in: Kritische Justiz 2010, S. 320 ff. Schriftleitergesetz vom 4.10.33: M. Hirsch, D. Majer, J. Meinck (Hg.), Recht, Verwaltung und Justiz im Nationalsozialismus. 2. Aufl. 1997, S. 229 ff. Walter Mallmann: Heinrich Beck 1963, S. 182, 195, 201; W. Schmitt, Walter Mallmann, in: B. Diestelkamp, M. Stolleis (Hg.), Juristen an der Universität Frankfurt am Main, 1989 , S. 306 ff. Die $5^{2}$ Bücher zum NS-Recht nach der Bibliographie des Verlags 1913-1988. Wolfgang Hefermehl: SS-Akte im Bundesarchiv Berlin Sign.ZB 5252; zur Behandlung des Feindvermögens: Stephan H. Lindner, Das Reichskomissariat für die Behandlung feindlichen Vermögens im Zweiten Weltkrieg, 1991, das Zitat S. 5. Die 3 Artikel zur «Entjudung»: Deutsche Justiz 1938, S. 988 ff; 1938, S. 1981 ff.; 1941, S. 422 f.; im Übrigen: W. Knopp, Wolfgang Hefermehl, in: Juristen im Portrait, S. 396 ff.; P. Ulmer, Wolfgang Hefermehl (1906-2001), in: S. Grundmann, K. Riesenhuber (Hg.), Deutschsprachige Zivilrechtslehrer in Berichten ihrer Schüler, Bd.ı, 2007, S. 239 ff. Zu Hans Frank und der Akademie für Deutsches Recht: H.-R. Pichinot, Die Akademie für Deutsches Recht, 1981; H. Hattenhauer, Die Akademie für Deutsches Recht (1933-1944), in: Juristische Schulung, 1986, S. 68 off.

5. Stuckart, Globke: Alfred Flemming: H. Weber, Alfred Flemming und Walter Lewald, in: Juristen im Portrait, S. 325 ff.; seine Mitgliedschaft in der NSDAP: SA- oder NSSK-Akte Alfred Flemming Bundesarchiv Berlin. Wilhelm Stuckart: E. Klee, Das Personenlexikon zum Dritten Reich, 2. Aufl. 2007, S. 611 f. Hans Globke: J. Bevers, Der Mann hinter Adenauer, 2009; E. Lommatzsch, Hans Globke (1898-1973). Beamter im Dritten Reich und Staatssekretär Adenauers, 2009 (das bessere Buch); ein scharfer Angriff gegen ihn aus der DDR z. B.: H.-G. Cheim, A. Kutschke, P. Przybylski, Globke-Kriegsverbrecher und Verbrecher gegen die Menschlichkeit, in: Neue Justiz, 196o, S.719 ff. Otto Schwarz und Rechtsprechung des Reichsgerichts: F. K. Kaul, Geschichte des Reichsgerichts, Bd. 4, 1971 , S. 113 f., $326 \mathrm{f}$. 
Palandt: Das Zitat Helmut Heinrichs in Rechtswissenschaft und Literatur im 2o. Jahrhundert, S. 397. Otto Palandt: H. Heinrichs, Palandt-Der Mensch und das Werk, in: Ludwig-Maximilians-Universität München, Verleihung der Ehrendoktorwürde an Herrn Dr. h. c. Helmut Heinrichs, 1988, S. 18 ff.; H. Wrobel, Otto Palandt zum Gedächtnis ... in: Kritische Justiz 1982, S. 1 ff.; E. Barnert, Von Station zu Station. Anm. zu Otto Palandt (umstr) ..., in: myops 1, 2007, S. 56 ff. Gustav Wilke: Nachruf (anonym) in «Deutsche Justiz» 1938, S. 799. «Dr. rer. pol. und Dr.»jur» in: Gustav Wilke, Gewerblicher Rechtsschutz, 1936, Vahlen Berlin, Vorblatt. Allgemeine Annahme, Auftrag zum neuen Kommentar «Wilke/Palandt» 1934: Heinrich Beck 1963, S. 176; Helmut Heinrichs, Palandt Der Mensch und sein Werk, 1988, S. 21; Helmut Heinrichs, Bernhard Danckelmann, in: Juristen im Portrait, 1988, S. 231; Jan Albers, Wolfgang Lauterbach, Juristen im Portrait, 1988, S. 513; K. W. Slapnicar, Der Wilke, der später Palandt hieß, in: Neue Juristische Wochenschrift, 2000, S. 1694; besser: «Schnell» ... «wegen der Hitlerschen Rassengesetze»: Hans Dieter Beck 1988, S. 28. Loening, Basch, Straßmann 1934: Bibliographie Verlag C.H.Beck 1913-1988, 1988, S.65. Vertrag vom 31.8.1935 mit Loening, Basch, Straßmann über 2. Aufl.: Karl Schröpel, Georg Sund, Erklärung v. 18.7.1946 in der Entnazifizierungsakte Heinrich Beck, Blatt 97. Tagung der Reichsgruppe Hochschullehrer 3. und 4. Oktober: H. Göppinger, Juristen jüdischer Abstammung im «Dritten Reich», 1990, S. 154 ff., S. 154 die Erklärung Hans Franks. Das Datum 6. Dezember 1936 für den mit Wilke u. a. geschlossenen Verlagsvertrag: Helmut Heinrichs, Palandt, Bürgerliches Gesetzbuch, in: D. Willoweit (Hg.), Rechtswissenschaft und Rechtsliteratur im 20. Jahrhundert, 2007, S. 357 (Danckelmann starb erst 1981). Der Bericht über den Tod auf der Dienstfahrt: Akte des Reichsinnenministeriums, Dr. Franz Schlegelberger, Bundesarchiv Berlin, Schlegelberger am 6.8.1938 an Minister Gürtner, S. 192 f., Bundesarchiv Bestandssignatur R/30o1, Archivnummer 74179; A. Wulf, Staatssekretär Prof. Dr. Dr.h. c. Franz Schlegelberger, 1991, S. 43. Kein Mitglied in der NSDAP: Bundesarchiv Berlin, Mitgliederkartei der NSDAP, kein Eintrag, ebenso Angaben Schlegelbergers zur Ergänzung seines Personalbogens vom 5.8.41, Bundesarchiv Bestandssignatur R/3001, Archivnummer 74,179. Die Suche nach einem Nachfolger: K. W. Slapnicar, Neue Juristische Wochenschrift 2000, S. 1695 Anm.61. Alfred Flemming: H. Weber, Alfred Flemming und Walter Lewald, in: Juristen im Portrait, 1988, S. 325 ff. Mitglied der NSDAP seit 1.5.1933: Bundesarchiv Berlin, Mitgliederkartei der NSDAP. Rechtswahrer: C. Schmitz-Berning, Vokabular des Nationalsozialismus, 20oo, S. 530 f. Guido (von) List: Deutsche Biographische Enzyklopädie, hg. v. W. Killy und R. Vierhaus, Bd.6, 2001, S. 4.24 f. Zu Adolf Schönke siehe die Literaturhinweise bei XX. 15.

6. Andere Verlage im Vergleich: Mohr/Siebeck: S. Knappenberger-Jans, Verlagspolitik und Wissenschaft: der Verlag J. C. Mohr (Paul Siebeck) im frühen 2o. Jahrhundert, 2ool; U. Schneider, Der wissenschaftliche Verlag, in: E. Fischer, S. Füssel (Hg.), Geschichte des Deutschen Buchhandels im 19. und 20. Jahrhundert, Bd. 2, Teil 1, 2007, S. 408f. Carl Heymann: R. Schmidt, Deutsche Buchhändler. Deutsche Drucker, 1902 (Ndr. 1979), S. 443 ff; G. Jäger, Der wissenschaftliche Verlag, in: G. Jäger u. a. (Hg.), Geschichte des Deutschen Buchhandels im 19. und 20. Jahrhundert, Bd. 1, Teil 1, 2001, S. 493 ff. Franz Vahlen: R. Schmidt a. a. O. S. 501 ff. Junker und Dünnhaupt: Junker und Dünnhaupt: ein Almanach zum 10-jährigen Bestehen des Junker und Dünnhaupt Verlages, Berlin 19271937, 1937. Hanseatische Verlagsanstalt: S. Lokatis, Die Hanseatische Verlagsanstalt. Politisches Buchmarketing im «Dritten Reich», in: Archiv für die Geschichte des Buchwesens, 1992, S. 1-189.

\section{Westdeutsche Nachkriegszeit und ihr Buchhandel}

E. Umlauff, Der Wiederaufbau des Buchhandels. Beiträge zur Geschichte des Büchermarktes in Westdeutschland nach 1949, in: Archiv für die Geschichte des Buchwesens, Bd. 17, 1977/78, S. XVI ff und Sp.1-1750 (der ganze Band), dort das Militärregierungsge- 
setz Nr. 191 Sp. 1489 ff. und die Information Control Regulation No.1 Sp. 1491, jeweils englisch mit deutscher Übersetzung; B. R. Gruschka, Der gelenkte Buchmarkt. Die amerikanische Kommunikationspolitik in Bayern und der Aufstieg des Verlages Kurt Desch 1945 bis 1950, in: Archiv für die Geschichte des Buchwesens, Bd. 44, 1995, Sp. 1-182; $R$. Wittmann, Geschichte des deutschen Buchhandels, 1999, S. 392 f., 406 ff. Die in München 1945/46 lizensierten Verlage, meistens mit Datum der Lizenz nach E. Umlauff Sp.1683 ff., dort auch das von Biederstein, Hanfstaengl und Leibniz Verlag. Zu Ernst («Putzi») Hanfstaengl zuletzt P. Conradi, Hitlers Klavierspieler. Ernst Hanfstaengl - Vertrauter Hitlers, Verbündeter Rossevelts, 2007. Zum Verlag Hanfstaengl: R. Schmidt, Deutsche Buchhändler. Deutsche Drucker, 1902/1908 (Ndr.1979), S. 300 ff.; R. Wittmann, Hundert Jahre Buchkultur in München, 1993, S.52. Verlag Oldenbourg: R. Schmidt a. a. O. S. 729 ff.; R. Wittmann (1993) a. a. O. S. 119 f. Der Parteieintritt Alexander Oldenbourgs: B. R. Gruschka a. a. O. S. 139 Anm. 45o. Manfred Schröter: Deutsche Biographische Enzyklopädie, Bd.9, 2001, S. 156, seine Lizenz für den Leibniz Verlag: E. Umlauff a. a. O. S. 1691

\section{Entnazifizierungsverfahren gegen Heinrich Beck}

L. Niethammer, Die Mitläuferfabrik. Die Entnazifizierung am Beispiel Bayerns, 1982. C. Vollnhals, Entnazifizierung. Politische Säuberung und Rehabilitierung in den vier Besatzungszonen 1945-1949, 1991. Die Entnazifizierungsakte Heinrich Becks ist im Besitz seiner Söhne Hans Dieter und Wolfgang Beck; sie ist ferner im Staatsarchiv München SpkA K 102: Beck, Heinrich, Dr. Die Einstufung Wilhelm Stuckarts: L. Müller, Furchtbare Juristen, 1987 , S. 216 f.

\section{Aufarbeitung von Unrecht des Nationalsozialismus}

Adorno: T. W. Adorno, Eingriffe, 1965, S. 125. Zeitschrift RzW: H. Weber, Juristische Zeitschriften des Verlages C.H.Beck, 2007, S. 39ff. Ernst Féaux: E. Klee, Das Personenlexikon zum 3. Reich, 2. Auflage 2007, S. 145 Entschädigung: Georg Blessin/Hans-Georg Ehrig/Hans Wilden Bundesentschädigungsgesetze. Kommentar. 3. Aufl. 196o; Georg Blessin/Hans Gießler Bundesentschädigungsschlußgesetz. Kommentar. 1967; Blessin/ Hans Gießler/Hans Wilden Bundesrückerstattungsgesetz und elfte Verordnung über Ausgleichsleistungen nach dem Lastenausgleichsgesetz. Kommentar. 1958; Die Wiedergutmachung nationalsozialistischen Unrechts durch die Bundesrepublik Deutschland. Gesamtwürdigung in Einzeldarstellungen. Herausgegeben vom Bundesminister der Finanzen in Zusammenarbeit mit Walter Schwarz. Es erschienen sechs Bände ab 1974 und 1985 eine Schlußbetrachtung von Walter Schwarz.

\section{Berliner Niederlassung und Nördlinger Druckerei nach 1945}

A. Heinrich, Die C.H.Beck'sche Buchdruckerei Nördlingen in den Jahren 1945 bis 1979 , 1980, S. 1 ff.; E. Dilba, Typographie-Lexikon, 2. Aufl. 2008.

\section{Biederstein Verlag}

1. Heinrich Beck 1963, S. 189 f.; Gustav End, Grenzgang-Kreuzzwang, in: H. Proebst, K. Ude (Hg.), Denk ich an München, 1966, S. 286 ff.; o. Verf., Gustav End zum 7o. Geburtstag am 25.11.1970, C.H.Beck 1970; Hans Dieter Beck 1980, S. 4o f.; M. Wegner, Bayerisch und galant. Buchimperiums-Außenminister: Zum Tode von Gustav End, Frankfurter Allgemeine Zeitung 28.4.1994, S. 38; A. Heinrich, Gustav End t: Ein Wort des Dankes, in: Neue Juristisches Wochenschrift 1994, S. 1849 f.; Deutsche Biographische Enzyklopädie, Bd. 3, 2001, S. 107. Deutsche Buch-Gemeinschaft, Das Buch stiftet Gemeinschaft. Herausgege- 
ben im April 1964, zum vierzigjährigen Bestehen, 1964; Deutsche Buch-Gemeinschaft, 5o Jahre Deutsche Buch-Gemeinschaft, 1974.

2. Der Teilnachdruck des Palandt wird erwähnt von J. Albers, Wolfgang Lauterbach, in: Juristen im Portrait, S. 515. Carl Hoeller: Hans Dieter Beck 1988, S. 68 ff.

3. A. Flemming, Aus der Gründungsgeschichte der NJW, NJW 1987, S. 2653 ff.; H. Weber, Juristische Zeitschriften des Verlags C.H.Beck. Von den Anfängen im 19. Jahrhundert bis zum Zeitalter der elektronischen Medien, 2007, S. 15 ff.

4. Sonderausgabe des Palandt: J. Albers, Wolfgang Lauterbach, in: Juristen im Portrait, S. 514. Edmund Mezger: K. v. Jan, Juristen im Portrait, S. 561 ff. einerseits, E. Klee, Das Personenlexikon zum Dritten Reich, 2. Aufl. 2007, S. 409 f. andererseits. Vgl. auch noch R. v. Hippel, Moderne Wege der Strafrechtsdogmatik. Ergänzende Bemerkungen zum Lehrbuch des Strafrechts in seiner 3. Aufl. (1949), 1950. Besuch Gustav Ends beim Übersetzer von Nikolai Leskow: J. v. Guenther, Der junge Siebziger, in: o. Verf., Für Gustav End zum 70. Geburtstag am 25.11.1970, 1970, S. 20 ff.

5. Der Nachruf in der Frankfurter Allgemeinen vom 28. April 1994, S. 38.

\section{Bauarbeiten in München und in der Nördlinger Druckerei}

Roderich Fick: am besten der kleine Begleittext zu einer Ausstellung über ihn in Herrsching/Ammersee 2007, 158 Seiten, mit vielen Dokumenten und Abbildungen, wohl nur zu erhalten über das Archiv der Gemeinde Herrsching, «Roderich Fick. Baumeister in Herrsching» mit Beiträgen von Friederike Orth (seine Tochter), Friederike Hellerer (Leiterin des Archivs) und Thomas Harant, Dipl.Ing., Architekt. Bezeichnung als NS-Aktivist: H. Weihsmann, Bauen unterm Hakenkreuz, 1998, implizit an vielen Stellen, vgl. Index; E. Klee, Das Kulturlexikon zum Dritten Reich, 2007, S. 151; M. Krauss, Rechte Karrieren in München, 2010. Zur «Gottbegnadetenliste»: O. Rauskolb, Führertreu und gottbegnadet, 1991 (nicht zu Architekten). Zu Bautätigkeit und Druckerei: A. Heinrich, Die C.H.Beck'sche Buchdruckerei Nördlingen 1945 bis 1979 (auch zum Verlag); vgl. noch Heinrich Beck 1963, das Zitat dort S. 191; Zur Umstellung von Fraktur und Antiqua bei Beck: A. Heinrich a. a. O. S. 49, zum Führerbefehl 194ı: F. Beck, «Schwabacher Judenlettern». Schriftenverruf im Dritten Reich, in: Die Kunst das Vernetzens, Festschrift für Wolfgang Hempel, hg. v. B. Brachmann u. a., 2006, S. 251 ff.

\section{Die Bundesrepublik und ihr Buchhandel}

Allgemeine Geschichte: M. Görtemaker, Geschichte der Bundesrepublik Deutschland, 1999; derselbe Die Berliner Republik. Wiedervereinigung und Neuorientierung 2009. Wirtschaft: $W$. Abelshauser, Deutsche Wirtschaftsgeschichte seit 1945, 2004; E. Pies, Löhne und Preise von 1300 bis 2000, 6. Aufl. 2008. Buchhandel: R. Wittmann, Geschichte des deutschen Buchhandels, 3. Aufl. 2011, S. $39^{2}$ ff.; die Zahlen für die Neuerscheinungen/Erstauflagen der Bundesrepublik nach den Angaben der Veröffentlichung «Buch und Buchhandel in Zahlen» des Börsenvereins des deutschen Buchhandels jeweils im folgenden Jahr. Die juristischen Verlage mit ihren lieferbaren Titeln nach ihren Internetseiten, meistens unter ARSV (Arbeitsgemeinschaft der rechts- und staatswissenschaftlichen Verlage, http://www.juristische-verlage.de). Die von C.H.Beck aus dem Verlag.

\section{XIX: Heinrich Beck in der Bundesrepublik}

1. Heinrich Beck 1963, S. 256 ff.; Hans Dieter Beck 1988, S. 39 ff.

2. Die Zahlen entweder schon genannt oder errechnet nach der Bibliographie von 1988. 3. Hermann Weber, Juristische Zeitschriften des Verlages C.H.Beck, 2007, S. 27 ff., dort die Zitate S. 37 und 66; die Entscheidung des BGH zur NJW als «allgemein verwendete 
Wochenschrift» NJW 1979, S. 877. Uwe Diederichsen: NJW 1988, S. 1. Dessen Nietzsche Zitat: U. Diederichsen, Die BGB-Klausur, 1970, S. 173. Die Zahl der Entscheidungen in JR, JZ, MDR und NJW nach den Registern der Zeitschriften von 1970. Die Rechtsoziologie zum Wachsen des Rechtsstoffs, Gesetzes- und Prozessflut z. B. M. Rehbinder, Rechtssoziologie, 7. Aufl. 2oog, Rdz. 89 f.; 144, 159, dort Rdz. 90 Anm. 76 das Zitat von Alexander Hollerbach.

4. Hans Dieter Beck, 1988, S. zo f.; A. Heinrich 1980, S. $6 z$ ff.

5. Neues Bereicherungsrecht: E. v. Caemmerer, Bereicherung und unerlaubte Handlung, in: Festschrift Ernst Rabel, 1954, S. 333 ff.; BGHZ 40.272 (Elektroherdefall, 1963); Palandt/Thomas, BGB, 29. Aufl. 1970, Einf. 1 vor \$812; Pomponius Digesten 12.6.14; Palandt/Thomas, BGB, 31. Aufl. 1972, $\$ 812$ Anm. 1; Baumbach/Lauterbach: J. Albers, Wolfgang Lauterbach, in: Juristen im Portrait, 1988, S. 514. Böhle-Stamschräder: $W$. Uhlenbruck, Aloys Böhle-Stamschräder, in: Juristen im Portrait, 1988, S. 182 ff. Konrad Duden: H.-M. Pawlowski, G. Wiese, G. Wüst in der Festschrift für Konrad Duden, 1977, S.Vff.; H. Rowedder, Konrad Duden, in: Juristen im Portrait S. 270 ff. Thomas/Putzo: K. Reichold, Thomas/Putzo, Zivilprozessordnung, in: D. Willoweit (Hg.) a. a. O. S. 755 ff. 6. Otto Schwarz: C. Kirchner, Reichsgerichtsrat a. D. Dr. Otto Schwarz (Nachruf), in: NJW 1960, S.521 f.; H. Tröndle, Schwarz/Dreher/Tröndle/Fischer, Strafgesetzbuch, in: D. Willoweit (Hg.), Rechtswissenschaft und Rechtsliteratur im 20. Jahrhundert, 2007, S. 838 ff. Eduard Dreher: J. Müller, Furchtbare Juristen, 1987, S. 214 ff.; Karl Lackner, Eduard Dreher, in: Juristen im Portrait, S. 261 ff.; Dreher/Maassen: Hans Dieter Beck, 1988, S. 33 .

7. Maunz: P. Lerche, Theodor Maunz in: Juristen im Portrait, S. 553 ff., der Aufsatz von $K$. Redeker, Bewältigung der Vergangenheit als Aufgabe der Justiz, NJW 1964, S. 1097 ff.; zur Arbeit für Gerhard Frey: M. Stolleis, Theodor Maunz. Ein Staatsrechtslehrerleben, Kritische Justiz, 1993, S. 393 ff. Dürig: H.-U. Büchting, in: Juristen im Portrait, S. 28 o ff.; Dürig als Architekt: $P$. Lerche, Günter Dürig als Architekt, in: Peter Lerche u. a., Zum Gedenken an Prof. Dr. iur. Günter Dürig (1920-1996), 1999, S. 1 ff., im Übrigen mündliche Auskünfte von Dieter Burneleit, Ingo von Münch und Walter Rudolf. Maunz/Dürig: P. Lerche, Maunz/Dürig, in: Rechtswissenschaft und Rechtsliteratur S. 109 ff. Folter im Rechtsstaat: zur «Isolationsfolter» der RAF z. B. S. Cobler, Die Gefahr geht von den Menschen aus, 1976, S. 100 ff.; die Beschlüsse des BGH und BVerfG: BGH NJW 76 .III und BVerGE 41.246. Eine sehr gute und genaue Beschreibung der Folterdiskussion von Ernst Albrecht bis Matthias Herdegen mit dem Fall Daschner: J.P. Reemtsma, Folter im Rechtsstaat?, 2005. Die beiden Aufsätze von Brugger in: Der Staat 1996, S. 67 ff. und JZ 2000. 165 ff. Matthias Herdegen zuletzt: Maunz/Dürig, 2005, Art. 1 Abs. 1, Rdz. 43 ff., noch einmal 2oo6, Rdz. 9o. Das Urteil gegen Wolfgang Daschner: LG Frankfurt/Main, NJW 2005, S. 692 ff. Im Übrigen: E.-W. Böckenförde, Die Menschenwürde war unantastbar, FAZ 3.9.2003, S. 33 und 35. Die drei anderen Kommentare zu Herdegen/Brugger: C. Starck in: von Mangoldt/Klein, 6. Aufl. 2010, Art. 1 Abs. 1, Rdz.79; H. Dreier in: Dreier, 2. Aufl. 2004, Art. 1 Abs. 1, Rdz. 133 unter Hinweis auf seinen Schüler Fabian Wittreck in Anm. 437; P. Kunig in: von Münch/Kunig, 6. Aufl. 2011 , Art. 1, Rdz. 36 «Folterverbot».

8. a) Grüne Lehrbücher: Ernst Forsthoff: H. Schneider, Ernst Forsthoff: 70 Jahre, in: Neue Juristische Wochenschrift, 1972, S. 1654; K. Doehring, Ernst Forsthoff, in: Juristen im Portrait, S. 34,1 ff.; H. Quaritsch, Erinnerung an Ernst Forsthoff, in: Neue Juristische Wochenschrift, 1974, S.2120; G. Mauz, Ernst Forsthoff und andere, in: K. Korino, E. Jaeckel (Hg.), Intellektuelle im Bann des Nationalsozialismus, 1980, S. 193 ff.; S. 197 f. der Hinweis auf Artikel im «Deutschen Adelsblatt»; M. Stolleis, Verwaltungswissenschaft in der Bundesrepublik Deutschland, in: D. Simon (Hg.), Rechtswissenschaft in der Bonner Republik, 1994, S. 235; F. Hermann, Forsthoff, Ernst, in: M. Stolleis (Hg.), Juristen, 1995, S. 212 ; B. Rüthers, Überlebende und überlebte Vergangenheit, in: myops, 2oo8, S. 67 ff.; F. Meinel, Der Jurist der industriellen Gesellschaft. Ernst Forsthoff und seine Zeit, 2011; M. Stolleis, Geschichte des öffentlichen Rechts in Deutschland, 4. Band, 2012, S. $178 \mathrm{ff}$. 
Karl Larenz: U Diederichsen, Karl Larenz, in: Juristen im Portrait, S. 495 ff., das Zitat S. 509; C.-W. Canaris, Karl Larenz (Nachruf), in: Juristenzeitung 1993, S. 404,ff., H. Köhler, Karl Larenz (Nachruf), in: Versicherungsrecht 1993, S. 420 f.; R. Dreier, Karl Larenz über seine Haltung im «Dritten Reich», in: Juristenzeitung 1993, S. 454,ff. (Larenz' Brief an Dreier von 1987); H. H. Jakobs, Karl Larenz und der Nationalsozialismus, in: Juristenzeitung 1993, S. 805 ff.; J. Prölss, Erwiderung auf Jacob's Beitrag zu Karl Larenz, in: Juristenzeitung 1994, S. 33 f.; H. H. Jakobs, Schlusswort, in: Juristenzeitung 1994, S. 34; $R$. Frassek, Von der «völkischen Lebensordnung» zum Recht. Die Umsetzung weltanschaulicher Programmatik in den schuldrechtlichen Schriften von Karl Larenz (1903-1993), 1996; B. Rüthers, Die unbegrenzte Auslegung, 5. Aufl. 1997, S. 123 ff.; R. Frassek, Karl Larenz (1903-1993)-Privatrechtler im Nationalsozialismus und im Nachkriegsdeutschland, in: Juristische Schulung 1998, S. 296 ff.; E. Klee, Das Personenlexikon zum Dritten Reich, 2. Aufl. 2007, S. 358; B. Hüpers, Karl Larenz - Methodenlehre und Philosophie des Rechts in Geschichte und Gegenwart, 2010, das «krasse» Urteil S. 269; C.-W. Canaris, Karl Larenz, in: K. Riesenhuber, S. Grundmann (Hg.), Deutschsprachige Zivilrechtslehrer in Berichten ihrer Schüler ..., Band 2, 2010, S. 264 ff.; B. Rüthers, Personenbilder und Geschichtsbilder - Wege zur Umdeutung der Geschichte. Anmerkungen zu einem LarenzPortrait, in: Juristenzeitung 2011, S. 593 ff.; das erste Zitat von Larenz 1935 bei Canaris: Karl Larenz, Rechtsperson und subjektives Recht, 1935, S. 21, das zweite Zitat S. 22, vgl. das Parteiprogramm der NSDAP von 1920 bei K. Pätzold, M. Weißbecker, Geschichte der NSDAP 1920 bis 1945, 3. Aufl. 2009 , S. 46 ff., Punkt 4 und 5 auf S. 46 ; C. - W. Canaris, «Falsches Geschichtsbild von der Rechtsperversion im Nationalsozialismus» durch ein Portrait von Karl Larenz? in: Juristenzeitung 2011, S. 879 ff.; B. Rüthers, Die Risiken selektiven Erinnerns-Antwort an C.-W. Canaris, in: Juristenzeitung 2011, S. 1149 ff.; D. Simon, Des Teufels Advokat, in: myops 12,2011, S. 65 ff.; H. H. Jacobs, Sehr geehrter Herr Canaris, in: myops 14, 2012, S. 6 ff.; J. Kaube, Der Fremde als Gast im Zivilrecht, in: Frankfurter Allgemeine Zeitung 11. April 2012, Seite N3 («Geisteswissenschaften»). Fritz Baur: H. Schultze-v. Lasaulx, Vergleichende Rezension, in: Juristenzeitung 1963, S.612 ff.; M. Wolf, Fritz Baur, in: Juristen im Portrait, S. 139 ff.; U. Diederichsen, W. Grunsky, R. Stürmer, H. P. Westermann, zum Gedenken an Prof. Dr.iur. Fritz Baur, 1994; A. Thier, Sachenrecht, in: Rechtswissenschaft und Rechtsliteratur, S.264ff.; Reichserbhofgesetz: $M$. Hirsch, D. Majer, J. Meinck, Recht, Verwaltung und Justiz im Nationalsozialismus, 2. Aufl. 1997, S. 388 f. Heinrich Lange: D. Leipold, Erbrecht, in: Rechtswissenschaft und Rechtsliteratur S. 357 ff. Joachim Gernhuber: D. Schwab, Familienrecht, in: Rechtswissenschaft und Rechtsliteratur, S. $33_{2}^{2}$ ff.; $U$. W., Der Gang nach Karlsruhe, 2004, S. 151 ff.

b) Kurz-Lehrbücher: Theodor Maunz (Konrad Hesse, Ekkehart Stein): W. Pauly, Verfassungs- und Verfassungsprozeßrecht, in: Rechtswissenschaft und Rechtsliteratur, S. 922 f; M. Stolleis, Geschichte des öffentlichen Rechts in Deutschland, 4. Band 2012, S. $488 \mathrm{ff}$. Günther Beitzke: F. Gamillscheg, Günther Beitzke, in: Juristen im Portrait, S. 147 ff., das Zitat S. 154; D. Schwab, Familienrecht, in: Rechtswissenschaft und Rechtsliteratur, S. 330 ff., das Zitat S. 331. Friedrich Lent: W. Habscheid, Friedrich Lent, in: Juristen im Portrait, S. $5^{21}$ ff. Erich Molitor: H. Schlosser, Molitor, Erich, in: Neue Deutsche Biographie, Band 17, 1997, S. 726 f. Heinrich Lange: K. Kuchinke, Heinrich Lange (Nachruf), in: Neue Juristische Wochenschrift, 1978, S. 309; E. Klee, Das Personenlexikon zum Dritten Reich, 2. Aufl. 2007, S. 356. Der Begriffshimmel: Rudolfv. Jhering, Scherz und Ernst in der Jurisprudenz, 1884, Überschrift der 3. Abteilung, auch in späteren Auflagen. Die «Crux» bei F. Wieacker, Privatrechtsgeschichte der Neuzeit, 2. Aufl. 1967, S. 488; zum Problem des Allgemeinen Teils: $U$. Diederichsen, Allgemeiner Teil des BGB, in: Rechtswissenschaft und Rechtsliteratur, S. 93 f., zum Buch von H. Lange S. 94 f. Horst Bartholomeyczik: W. Harms, Horst Batholomeyczik (Nachruf), in: Neue Juristische Wochenschrift 1975, S. 1550; E. Klee, Das Personenlexikon des Dritten Reichs, 2. Aufl. 2007, S. 29; I. Heinemann, «Rasse, Siedlung, deutsches Blut». Das Rasse- und Siedlungshauptamt der SS und die rassenpolitische Neuordnung Europas, 2. Aufl. 2003, S. 135 (dort die Meldung vom Dezember 
1940), 150 Anm. 75/610; zum Bodenamt in Prag S. 131 ff. Hans Julius Wolff: M. Kriele, Hans J. Wolff, in: Juristen im Portrait, S. 694 ff.; derselbe, Hans J. Wolff (Nachruf), in: Neue Juristische Wochenschrift, 1977, S. 28 f. U. Battis, Ein deutscher Staatsrechtler in der NS-Zeit, in: Neue Juristische Wochenschrift, 1939, S. 884 f.; M. Stolleis a. a. O. 3. Band, 1999, S. 266, und 4. Band, 2012, S. 184 f.; A. Voßkuhle, Allgemeines Verwaltungs- und Verwaltungsprozeßrecht, in: Rechtswissenschaft und Rechtsliteratur, S. 957 f.; Zur Auslandsorganisation der NSDAP allgemein: $V$. Koop, Hitlers fünfte Kolonne, 2009.

9. Rechtsgeschichte: Paul Koschaker: G. Ries, Koschaker, Paul, in: Neue deutsche Biographie, Bd. 12, 1980, Sp. 608 ff.; Churchils Rede: G. Brunn, Die europäische Einigung, 3. Aufl. 2009, S. 7 ff., Europarat, dort S. 51 ff. William Seagle: Kritik aus der Rechtsanthropologie z. B. E. A. Hoebel, Rezension Seagle, in: American Anthropologist, 194,2, S. 14,1 ff. Max Kaser: die beste Beschreibung seines Hauptwerks ist wohl: T. Giaro, Max Kaser (1906-1997), in: Rechtshistorisches Journal Bd. 16, 1997, S. 231-357.

\section{Hans Dieter Beck in der Bonner Republik}

1. Hans Dieter Beck: Hans Dieter Beck 1988, S. 4,1; H. D. Beck, Neue Entwicklungen des Verlagsunternehmens, in: Rechtswissenschaft und Literatur, S. 1192 ff.; H. D. Beck, Verlagserwerbungen, in: Rechtswissenschaft und Rechtsliteratur, S. 1202 ff.

2. Vahlen: Allgemein R. Schmidt, Deutsche Buchhändler. Deutsche Buchdrucker, 2. Bd. 1903, Ndr. 1979, S. 351; Hans Dieter Beck 1988, S. 41. Walter Blümich: M. Friedenberger, Walter Blümich, in: M. Friedenberger, K.-D. Gössel, E. Schönknecht (Hg.), Die Reichsfinanzverwaltung im Nationalsozialismus, 2002 , S. $260 \mathrm{ff} . ;$ M. Friedenberger, Fiskalische Ausplünderung, 20o8, S. 19, 42, 159, 162 ff., 171, 321, 351 f., 385. Franz Schlegelberger: M. Förster, Jurist im Dienst des Unrechts. Leben und Werk des ehemaligen Staatssekretärs im Reichsjustizministerium Franz Schlegelberger, (1876-1970), 1995; L. M. PeschelGutzeit (Hg.), Das Nürnberger Juristen-Urteil von 1947, 1996. Günter Wöhe: Nachruf in: Börsen-Zeitung, 4.1.2008, S.7; Der Spiegel, 14.1.2008, S. 14,2; D. Gräbner, «Der Wöhe» macht ihn unvergessen, Saarbrücker Zeitung, 23.2.20o8, Spezial/Momente West.

3. und 4. Münchener Kommentare: Hans Dieter Beck 1988, S. 44ff.; U. Diederichsen, Münchener Kommentar zum BGB, in: Rechtswissenschaft und Rechtsliteratur, S. 109 ff.; F. J. Säcker, Münchener Kommentar zum BGB, in: Rechtswissenschaft und Rechtsliteratur S. $405 \mathrm{ff}$.

5. Eine Million BGB-Texte im dtv: Abendzeitung München 17.11.1976, Seite 6. Othmar Jauernig: H.-B. Kim, W. Marschall v. Bieberstein (Hg.), Zivilrechtslehrer deutscher Sprache, 1988 , S. 182 .

6. Soziales Mietrecht: U.W., Geschichte des Rechts, 3. Aufl. 2oo6, S. 47 I f., S. 577 . Wolfgang Schmidt-Futterer: H. Blank, Wolfgang Schmidt-Futterer, in: Juristen im Portrait, S. 657 f. Arztrecht allgemein: T. Riegger, Die historische Entwicklung der Arzthaftung, Diss. Hamburg, 2007 , S. 152 ff. Adolf Laufs: B.-R. Kern, Prof. Dr. iur. Dr. iur. h. c. Adolf Laufs zum 7o. Geburtstag, in: Medizinrecht, Jg. 2005, S. 621 ; Festschrift Adolf Laufs, 20o6, S. V ff. Nichteheliche Lebensgemeinschaft, Entwicklung: $C$. Schreiber, Die nichteheliche Lebensgemeinschaft, 1995, S. 6 ff.; U. W. Geschichte des Rechts, 3. Aufl. 20o6, S. 573. Siegfried de Witt und Johann-Friedrich Huffmann: mündliche Auskunft von JohannFriedrich Huffmann, Berlin.Murad Ferid: H. J. Sonnenberger, Murad Ferid, in: Juristen im Portrait, S. 311; P. Lichtenberger, Murad Ferid zum 9o. Geburtstag, in: Neue Juristische Wochenschrift, 1998, S. 1129 ; derselbe, Nachruf auf Murad Ferid, in: Neue Juristische Wochenschrift 1999, S. 196. Karl Firsching: D. Henrich, Karl Firsching, in: Juristen im Portrait, S. 321 ff.; H.-B. Kim, W. Marschall v. Bieberstein a. a. O. (oben Nr.6), S. 96 f.

7. Zum Wettbewerbs- und Immaterialgüterrecht: H. Köhler, Lauterkeitsrecht, und $U$. Loewenheim, Immaterialgüterrecht, in: Rechtswissenschaft und Rechtsliteratur, S. $5^{2} 9 \mathrm{ff}$. und S. $55^{1} \mathrm{ff}$. 
8. Otto Rudolf Kissel: W. Hiersemann, Neuer Präsident des BAG: Dr. Otto Rudolf Kissel, in: Neue Juristische Wochenschrift, 1981, S. 1084f.; H. Wißmann, Otto Rudolf Kissel zum 75. Geburtstag, in: Neue Zeitschrift zum Arbeitsrecht, 2004, S. 30.

9. Handbücher: D. Willoweit, Juristische Literatur des 2o. Jahrhunderts, in: Rechtswissenschaft und Rechtsliteratur, S. 54 ff. Römische Kauklasjurisprudenz: F. Wieacker, Römische Rechtsgeschichte, 1. Abschnitt, 1988, S. 570. Formularsammlungen im Mittelalter: G. Schmitz, Formel, Formulare, Formelsammlung, in: Handwörterbuch zur deutschen Rechtsgeschichte, Bd. 1, 2. Auflage, 2008, Sp. 1616 ff.

10. Arbeitsrecht: Günter Schaub: P. Hanau, Dr. h. c. Günter Schaub, in: Neue Zeitschrift für Arbeitsrecht (NZA) 1994, S.741 f.; M. Schlachter, R. Ascheid, H.-J. Friedrich, Geleitwort, in: dieselben (Hg.), Festschrift für Günter Schaub, 1998, S. VII f.; M. Schlachter, Dr. h. c. Günter Schaub zum 7o. Geburtstag, in: NZA 2003, S. 32; P. Hanau, Ein Leben für das Arbeitsrecht - Günter Schaub zum 70. Geburtstag, in NZA 2003, S. 122; M. Schlachter, Dr. h. c. Günter Schaub zum 75. Geburtstag, in: NZA 2008, S. 29. Reinhard Richardi: ohne Verfasser, Professor Dr. Reinhard Richardi zum 65. Geburtstag, in: NZA 2002, S. $289, B$. Rüthers, Reinhard Richardi 70 Jahre, in: Recht der Arbeit 2007, S. 185 f. P. Hanau, Reinhard Richardi 70 Jahre, in: NZA 2007, S. 289; G. Annaß, Reinhard Richardi zum 75. Geburtstag, in: NZA 2012, S. 311 . Albert Gnade: BAG: «Dienstältester» ehrenamtlicher Richter - Albert Gnade wirkte 36 Jahre lang an BAG-Rechtsprechung mit, in: Der Betrieb 2001, Heft 19, S. 20. Wolfgang Däubler: mündliche Auskunft. Karl Fitting: F. Auffarht, Nachruf auf Karl Fitting, in: Neue Juristische Wochenschrift 1990, S. 2866 und H. Wi $\beta$ mann, Karl Fitting, in der Zeitschrift soziales Recht 2013, S. (das Manuskript hat er mir vorher überlassen).

11. Sozialrech: B. Schulte, Sozialrecht, in: Rechtswissenschaft und Rechtsliteratur, S. 647 ff.; O.E. Krasney, Kasseler Kommentar Sozialversicherungsrecht, a. a. O. S. 581 ff.; J. Meyer-Ladewig, Meyer-Ladewig/Keller/Leitherer, Sozialgerichtsgesetz, a. a. O. S. 691 ff.; B. Rüthers, Geschichte des Sozialrechts in Deutschland, 2003, S. $307 \mathrm{ff}$.

12. Verfassungsrecht: von Münch/Kunig: $W$. Pauly, Verfassung und Verfassungsprozeßrecht, in Rechtswissenschaft und Rechtsliteratur, S. 925; Jarass/Pieroth: Pauly a. a. O., S. 925; H. Zacher, Rezension, in: Juristenzeitung 1990, S. 36 . Badura, Staatsrecht: Pauly a. a. O. S. 923; F. Ossenbühl, Rezension, in: Der Staat, Bd. 26, 1987, S. 449 ff.; I. von Münch, Rezension, in: Neue Juristisch Wochenschrift, 2000, S. 1171. Klaus Stern: W. Frotscher, Rezension von Band V, in: Die Verwaltung, 2002, S. 291 ff.; W. Pauly, Verfassungs- und Verfassungsprozeßrecht, in: Rechtswissenschaft und Rechtsliteratur, S. 919 ff.; C. Starck, Rezension von Band IV.2, in: Juristenzeitung 2011, S. 623 f.; Zur Diskussion über Art. 3 Abs. 2 GG im Parlamentarischen Rat: M.F. Feldkamp, Der Parlamentarische Rat 194.81949, überarbeitete Neuausgabe, 2008, S. 69 f., 73 f. Zu Elisabeth Selbert: B. Meyer, Elisabeth Selbert (1896-1986), in: Kritische Justiz (Hg.), Streitbare Juristen, 1988, S. 427 ff. und M. Röwekamp, Juristinnen, 2005, S.395 ff. Selbst W. Frotscher, B. Pieroth nennen Frau Selbert in ihrer kurzen Verfassungsgeschichte, II. Aufl. 20I2, S. 38I, Rdz. 787 .

13. Verwaltungsrecht: Zu Eyermann/Fröhler, Ferdinand Kopp und den Großkommentaren: A. Voßkuhle, Allgemeines Verwaltungsrecht, in: D. Willoweit (Hg.) a. a. O., S. 964ff.; W.R. Schenke, Kopp/Schenke, Verwaltungsgerichtsordnung, in: Rechtswissenschaft und Rechtsliteratur, S. 1027 ff.; A. Grundei, Rezension, in: Neue Juristische Wochenschrift, 1977, S.712. S.Detterbeck, Ferdinand O. Kopp (Nachruf), in: NJW 1995, S. $2771 \mathrm{f}$.

14. Europarecht allgemein: R. Wolfrum, Völkerrecht, in: Rechtswissenschaft und Rechtsliteratur, S. 1053 ff; dort S. 1061 ff. C. Callies. Eberhard Grabitz: R. Scholz, Nachruf auf Eberhard Grabitz, in: NJW 1993, S. 712 f.

15. Strafrecht: H.Tröndle, Schwarz/Dreher/Tröndle/Fischer, Strafgesetzbuch, in: Rechtswissenschaft und Rechtsliteratur, S. 835 ff; Herbert Tröndle: Herbert Tröndle, in: E. Hilgendorf (Hg.), Die deutschsprachige Strafrechtswissenschaft in Selbstdarstellungen, 2010, S. 595 ff. Thomas Fischer: seine website im Internet unter http:/fischer-stgb.de/2. 
hzml, Stand 24.9.2011, die Rezension der 50. Auflage, «Fischer, StGB» 2001: M. Hettinger, in: Neue Juristische Wochenschrift, 2001, S. 3215 f., das Zitat S. 3252. Karl Lackner: Karl Lackner, in: E. Hilgendorf a. a. O. S. 269 ff. Hermann Maassen: Munzinger Archiv Personen im Internet unter Biographie, Maassen, Hermann, www.munzinger.de, Stand 23.9.2012. Kristian Kühl: website Dr.iur Dr.phil.Dres. h. c. Kristian Kühl unter http:/www. jura-uni-tuebingen.de/professoren_und_dozenten. Zur Entwicklung des «Schönke/ Schröder»: A. Eser, Schönke/Schröder, Strafgesetzbuch, in: Rechtswissenschaft und Rechtsliteratur, S. 85ı ff. Adolf Schönke: $G$. Wendt, Adolf Schönke, in: Juristen im Portrait, S. 663 ff.; A.-M. v. Lösch, Der nackte Geist. Die juristische Fakultät der Berliner Fakultät im Umbruch von 1933, 1999, S. 34 off. E. Klee, Das Personenlexikon zum Dritten Reich, 2. Aufl. 2007 , S. 556; A. Esser a. a. O. S. 851 ff. Horst Schröder: E. Dreher, Zum Tode Horst Schröders, in: Juristische Rundschau, 1973, S.461 f.; T. Lenckner, Zum Tode von Horst Schröder, in: Juristenzeitung, 1973, S. 799 f.; P. Cramer, Horst Schröder, in: Juristen im Portrait, S. 67 I ff.; $W$. Stree, Horst Schröder, in: Juristen im Portrait, S. 67 I ff.; W. Stree, Horst Schröder - Leben und Werk 1913-1973, in: Gedächtnisschrift Horst Schröder, 1978, S. 1 ff. A. Eser, Neue deutsche Biographie, Bd. 23, 2007, S. 566. Ulrich Eisenberg: mündliche Auskünfte meines Kollegen U.E. an der FU Berlin.

17. Der «Schmidt»: Zur Geschichte des Kommentars: L. Schmidt, Schmidt, Einkommenssteuergesetz, in: Rechtswissenschaft und Rechtsliteratur, S. 1119 ff., dort S. 1119 das Zitat. Ludwig Schmidt: L. Drenseck, Ludwig Schmidt zum Fünfundsiebzigsten, in: Deutsches Steuerrecht, 2003, S. 1461; F. Wassermeyer, Ludwig Schmidt 75 Jahre alt, in: Der Betrieb, 2003, Heft 34, S. 1; W. Schön, Nachruf, in: Finanzrundschau 2011, S. $1125 ; H$. Weber-Grellet, Nachruf, in: Deutsches Steuerrecht, 2011, S. 2117.

18. Die neuen juristischen Fakultäten/Fachbereiche: $M$. Stolleis, Geschichte des öffentlichen Rechts in Deutschland, 4. Band, 2012, S. 409. Studentenzahlen 196o-9o: H.-U. Wehler, Deutsche Gesellschaftsgeschichte, 5. Band, 2008, S. 380 f. Günther Schwerdtfeger: zum Buch knapp M. Stolleis a. a. O., zur Person: seine Website im Internet der Universität Hannover vom 28.10.2012. Hartmut Maurer: $P$. Häberle, Hartmut Maurer zum 70. Geburtstat, NJW 2001, S.736f.; A. Voßkuhle, Allgemeines Verwaltungsrecht, in: Rechtswissenschaft und Rechtsliteratur S.96of. Geschichte des informalen Verwaltungshandelns kurz mit weiterführender Literatur U. W., Geschichte des Rechts, 3. Auflage 2006, S. 565 f. Helmut Köhler: Uwe Diederichsen, in: Rechtswissenschaft und Rechtsliteratur, S. 98 f.; im Übrigen Lebenslauf und Schriftenverzeichnis im Internet auf seiner Website der Universität München, 28. Oktober 2012. Bernd Rüthers: Website der Universität Konstanz, 30. Oktober 2012. Dieter Medicus: $V$. Beuthien, Dieter Medicus zum 70. Geburtstag, NJW 2004, S. 1642; M. Fuchs, Dieter Medicus zum 80. Geburtstag, NJW 2009, S. 1400 f.

19. Max Kaser: D. Medicus, Max Kaser, in: Juristen im Portrait, S. 147 ff.; T. Giaro, Max Kaser (1906-1997), in: Rechtshistorisches Journal, 1997, S. 231-357, das Zitat S. 240. W. Kunkel, Römische Rechtsgeschichte, 12. Auflage, 1990, S. 92 f. Dieter Willoweit, seine Website der Juristischen Fakultät Würzburg, 15. Oktober 2012. Heinrich Mitteis: $G$. Landwehr, Heinrich Mitteis, in: Juristen im Portrait, S. 572 ff., dort Anmerkung 2 und 10 die Zitate von Feine und Bader; G. Diestelkamp, Drei Professoren der Rechtswissenschaft in bewegter Zeit ..., 200o, S. 9 ff. Heinz Lieberwirth: R. Heydenreuther, Nachruf, NJW 2000, S. 933 f. Wende im mitteleuropäischen Mittelalter: U.W., Geschichte des Rechts in Europa, 2012, S. 180 ff. Germanenproblem: R. Ogorek, Rechtsgeschichte in der Bundesrepublik, 1994, S. 6 off. Hinrich Rüping: seine Website der Kanzlei Dr. Rüping und Partner, Hannover, 15. Oktober 2012. Günter Jerouschek: website Rechtswissenschaftliche Fakultät der Universität Jena, Prof. Dr. iur., Dr. phil., Dr. iur. h. c. Günter Jerouschek, MA. Helmut Coing: K. Luig, Helmut Coing, in: Juristen im Portrait, S. 215 ff.; M. Stolleis, Rezension Europäisches Privatrecht, in: Rechtshistorisches Journal Bd.9, 199o, S. 81 ff.; M. Brauneder, Rezension Europäisches Privatrecht, in: Zeitschrift für neuere Rechtsgeschichte, 1993, S. 225 ff.; R. Ogorek, Rechtsgeschichte in der Bundesrepublik 
(1945-1990), in: D. Simon (Hg.), Rechtswissenschaft in der Bonner Republik, 1994, S. 12 ff., 54 f., 88 f. D. Simon, zwischen Wissenschaft und Wissenschaftspolitik: Helmut Coing (28.2.1912-15.8.2000), in: NJW 2001, S. 1029 ff. B. Döhlemeyer, Rechtsgeschichte, in: Rechtswissenschaft und Rechtsliteratur, S. 1147 ff., 1153 . Walter Wilhelm: D. Simon, Walter Wilhelm (1928-2002). Eine Erinnerung, in: Rechtsgeschichte, Bd.2, 2003, S. 142 ff. Michael Stolleis: seine Website am Max-Planck-Institut für europäische Rechtsgeschichte 21. Oktober 2012. Rezensionen: 1. Band: G. Roellecke, Juristenzeitung 1939, S. 291; D. Wyduckel, NJW 1989, S. 2455 f.; O. Kimminich, Die Öffentliche Verwaltung 1989, S. 827; A. Blankenagel, Kritische Justiz 1989, S. 365 ff. 2. Band: G. Roellecke, Juristenzeitung 1993, S. 727; O. Kimminich, Die Öffentliche Verwaltung 1994, S. 747 f. z. Band: M.H. Wiegandt, Kritische Justiz, 2000, S. 651 ff., das Zitat S.66o. 4. Band: C. Hillgruber, Doch den Beleuchter sieht man nicht, Frankfurter Allgemeine Zeitung, 13. August 2012, S. 10.

2o. Zeitschriften: Alles ausführlich beschrieben von Hermann Weber in seinem Buch «Juristische Zeitschriften des Verlages C.H.Beck», 2007, S. 131 ff.

\section{Deutschlands Vereinigung und Zerfall des Ostblocks}

Wiedervereinigung: M. Görtemaker, Geschichte der Bundesrepublik Deutschland, 1999, S. 715 ff.; M. Görtemaker, Die Berliner Republik, 2009, S. 7 ff.; Zitat Hobsbawm: E. Hobsbawm, Das Zeitalter der Extreme, dtv, 8. Aufl. 2007, S.592; Russland, Polen, Tschechien: U. W., Geschichte des Rechts in Europa, 2010 , S. 546 ff.; 560 ff. Rumänien: A. U. Gabanyi, Systemwechsel in Rumänien, 1998, S. 165 ff. Rechtsstaatbegriff: $U . W$., Geschichte des Rechts, 3. Aufl. S. 434, und Literatur zu Rdz. 273, S. 490

\section{Hans Dieter Beck in der Berliner Republik 1990-2013.}

H.D. Beck, Neue Entwicklungen des Verlagsunternehmens, in: Rechtswissenschaft und Literatur, S. 1192 ff.; H. D. Beck, Verlagserwerbungen, in: Rechtswissenschaft und Rechtsliteratur, S. 1202 ff.; D. Willoweit, Juristische Literatur des 20. Jahrhunderts in: Rechtswissenschaft und Rechtsliteratur, S. 54 ff.; Zahlen der Rechtsanwälte von 1880 bis 2000 bei $U$.W. Risiko Rechtsanwalt, 2001, S. 57 ff., bis 2009: NJW Dokumentation Heft 13, 2009 , S. XXXII. 